\title{
A two-stage method for assessing the efficiency of the three-stage series network data envelopment analysis model with two feedback
}

\author{
Hamidreza Ghomi ${ }^{a}$, Morteza Rahmani ${ }^{a, b^{*}}$ and Morteza Khakzar Bafruei ${ }^{\text {a,b }}$
}

\begin{abstract}
andustrial engineering department, Research institute of Technology development (ACECR), Tehran, Iran
${ }^{b}$ University of Science and Culture, Tehran, Iran

\begin{tabular}{l}
\hline C H R O N I C L E \\
\hline Article history: \\
Received June 9, 2018 \\
Received in revised format: \\
August 10, 2018 \\
Accepted September 29, 2018 \\
Available online \\
September 29, 2018 \\
\hline Keywords: \\
NDEA \\
Three-stage series structure \\
Non-linear programming problem \\
Efficiency calculation \\
Linearization
\end{tabular}

A B S T R A C T

Data envelopment analysis models play an important role in decision making. In this paper, onestage and two-stage nonlinear programming problems are investigated in order to evaluate the efficiency of two types of network data envelopment analysis model. The first type of network data envelopment analysis model has a series structure with three stages and a feedback between the last step and the middle step, the second model has a three-stage series structure with two feedback between the final step and the first step and the middle step. By examining the overall efficiency of the models based on the one-stage programming problem, a two-stage programming problem is also applied in order to evaluate the efficiency of each step. In order to solve onestage nonlinear programming problems and two-stage linear and nonlinear programming problems derived from modeling, a linearization method based on coordinate transformation, and constant assumption and gradual growth of some variables is presented. In the last section, the proposed methods have been discussed using some numerical examples.
\end{abstract}

(C) 2018 by the authors; licensee Growing Science, Canada.

\section{Introduction}

The use of linear and nonlinear programming problems has always been considered since the emergence of these methods for modeling phenomena in management sciences and economics (Shenoy, 2007; Drury, 2006; Dantzig, 2016). One of the most important applications of these kinds of problems considered in recent years is to examine the efficiency in the context of the data envelopment analysis (DEA) (Cooper et al., 2004; Kao, 2014). During the last few years, a new type of programming problems known as two-stage linear or nonlinear programming problems has been considered to study the efficiency of DEA, and in various resources (Kao, 2006; Tavana \& Khalili-Damghani, 2014; Kao $\&$ Liu, 2003). Also, due to the complexity of existing models in real-world applications, a new type of the discussion in this field is introduced as the network DEA (NDEA) (Kao, 2014). The network data envelopment analysis with respect to modeling has various structures such as series, parallel, etc. The efficiency review of the models that have feedback has always been of interest among the researchers due to its common uses. For example, in (Liang et al., 2011), the efficiency of the two-stage model was investigated. Wang et al. (1997) and Seiford and Zhu (1999), were the first researchers who studied the

\footnotetext{
* Corresponding author.

E-mail address: rahmanimr@jdsharif.ac.ir (M. Rahmani)

(C) 2019 by the authors; licensee Growing Science, Canada. doi: $10.5267 /$ j.dsl.2018.9.001
} 
two-stage models. In these studies, the simple models that considered the output of the first stage as the input of the next stage were considered. Later this branch of the models was examined for various modes; For example, in a paper by Halkos et al. (2014), the categorization types of the two-stage models was investigated, which can be referenced to series models, dynamic network models, shared flow models, and static network DEA models. In their research, the linearization method which was used was based on setting the denominator equal to one. Despite the review of various two-stage models, a model with feedback has not been considered in that paper. Two-stage model with feedback was considered for the first time in a paper by Liang et al. (2011); in the model under consideration, the output of the second stage is considered as the input of the first stage.

The methods of calculating the efficiency of the two-stage models in various papers have been investigated; For example, Adam Shariff Adli et al. (2017) and Amirteimoori (2014) examined the DEA model for two-stage mode with undesirable outputs. In these papers, the series modes with discrete outputs for each stage were considered and the efficiency was examined. The auxiliaryvariables-based approach for calculating the efficiency of the two-stage envelopment analysis model in Ashrafi et al. (2011), was studied; also, the study of the efficiency of the two-stage models with consideration of fuzzy data in Nabahat (2015), was presented. Ashrafi et al. (2010) developed the Russell model to examine the efficiency of the two-stage models. Li et al. (2018) calculated the efficiency of the stages based on the arrangement of importance of each of the steps. Lim and Zhu (2018) used radial measure to test the efficiency. The two-stage models were used to calculate the efficiency of different centers, including Wanke and Barros (2014) and Fernandes et al. (2018), research. In these studies, a two-stage series model without considering feedback was applied to calculate the efficiency of bank centers. To solve this model, these researchers used the linearization method by setting the denominator equal one. Despotis et al. (2016) examined four types of two-stage models with different conditions without feedback.

In addition to the two-stage models, the three-stage models have been considered by various researchers. Kao (2017) examined the three-stage model under different modes; the models studied in his research included a three-stage model with a parallel structure and a three-stage model with a serial structure. In the three-stage models examined by Kao (2017), for two-stage parallel models and twostage series models, the discrete outputs and inputs were considered for each stage. Three-stage models in DEA have various applications, including the calculation of the efficiency of banks and industrial centers. For instance, Ebrahimnejad et al. (2014) used a three-stage model consisting of a series-parallel structure in order to examine the bank centers. The model under study in their research was without feedback and was solved by setting the denominator equal to one and transforming that to a linear model. In addition to the studies mentioned, we can refer to the paper by Shewell and Migiro (2016), which deals with the literature review of the three-stage models. In the examined article in three-stage mode, the feedback between the stages, as discussed in Liang et al. (2011), for two-stage mode, was not been studied. Also, other than studies by Liang et al (2011) and Li et al. (2018), the arrangement of the importance of the stages in other studies has not been included. Though, Li (2017) studied the arrangement of the importance of the stages; the two-stage programming problems were not been used in their research. Therefore, reviewing the three-stage model with feedback and presenting a two-stage programming model based on the importance of each of the stages is one of the innovations of this paper. Also, the review of these articles suggests that the nonlinear programming problem solving method based on linearization is based on setting the denominator equal to one method, which can add to the complexity of calculating the answer to the problem. To avoid this problem, in this paper a new linearization method based on the step-by-step motion has been applied.

In the second section of this article, the total performance of the three-stage model with a feedback between the final stage and the middle stage is examined. In the third section, the efficiency of a threestage model with a series structure with two feedback will be computed. Also, in these two mentioned sections, a new linearization method based on the assumption that some variables are constant and the 
gradual motion for linearization the problems under study is presented. The two-stage linear programming problem is presented to examine the efficiency of each compartment of two models in the fourth section, and the method for solving this branch of problems is in this section. In the fifth section, we will give some test problems for the subject discussed in the previous sections.

\section{The three-stage network envelopment analysis model with feedback in the middle stage}

According to Kao (2014), the performance of the three-stage model without feedback was studied. In this section, while studying the overall efficiency of the three-step model with a feedback in the middle stage (Fig. 1), we examine the efficiency of each of the stages.

$$
D M U_{j}, \quad j=1,2, \ldots, n,
$$

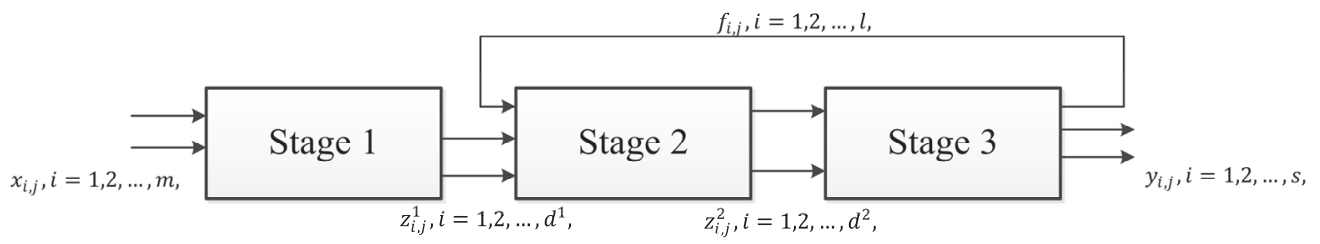

Fig. 1. Three stage model with feedback for the middle stage

In the first stage, we examine the effectiveness of each of the stages. For this purpose, we consider $\mathrm{DMU}_{\mathrm{j}}$ with value $\mathrm{j}=\mathrm{o}$ (o is an arbitrary value and $1 \leq \mathrm{o} \leq \mathrm{n}$ ). For this model, considering the inputs and outputs shown in Fig. 1, and without considering the relationships of the stages respect to each other, we examine the efficiency of each stage. So we calculate the efficiency of the first stage as follows:

$$
\begin{aligned}
& \max \frac{\sum_{\mathrm{i}=1}^{\mathrm{d}^{1}} \mathrm{~h}_{\mathrm{i}}^{1} \mathrm{z}_{\mathrm{i}, \mathrm{o}}^{1}}{\sum_{\mathrm{i}=1}^{\mathrm{m}} \mathrm{v}_{\mathrm{i}} \mathrm{x}_{\mathrm{i}, \mathrm{o}}}, \\
& \text { subject to } \frac{\sum_{\mathrm{i}=1}^{\mathrm{d}^{1}} \mathrm{~h}_{\mathrm{i}}^{1} \mathrm{z}_{\mathrm{i}, \mathrm{j}}^{1}}{\sum_{\mathrm{i}=1}^{\mathrm{m}} \mathrm{v}_{\mathrm{i}} \mathrm{x}_{\mathrm{i}, \mathrm{j}}} \leq 1, \mathrm{j}=1, \cdots, \mathrm{n} .
\end{aligned}
$$

$\mathrm{h}_{\mathrm{i}}^{1}, \mathrm{v}_{\mathrm{i}} \geq 0$

The efficiency of the second stage is calculated as follows:

$$
\begin{aligned}
& \max \frac{\sum_{i=1}^{d^{2}} h_{i}^{2} z_{i, o}^{2}}{\sum_{i=1}^{d^{1}} h_{i}^{1} z_{i, j}^{1}+\sum_{i=1}^{l} w_{i} f_{i, j}}, \\
& \text { subject to } \frac{\sum_{i=1}^{d^{2}} h_{i}^{2} z_{i, j}^{2}}{\sum_{i=1}^{d^{1}} h_{i}^{1} z_{i, j}^{1}+\sum_{i=1}^{l} w_{i} f_{i, j}} \leq 1, \quad j=1, \ldots, n .
\end{aligned}
$$

$\mathrm{h}_{\mathrm{i}}^{1}, \mathrm{~h}_{\mathrm{i}}^{2}, \mathrm{v}_{\mathrm{i}}, \mathrm{w}_{\mathrm{i}} \geq 0$

To evaluate the efficiency of the third stage, we present the following relation:

$$
\begin{aligned}
& \max \frac{\sum_{i=1}^{s} u_{i} y_{i, o}+\sum_{i=1}^{l} w_{i} f_{i, o}}{\sum_{i=1}^{d^{2}} h_{i}^{2} z_{i, o}^{2}}, \\
& \text { subject to } \quad \frac{\sum_{i=1}^{s} u_{i} y_{i, j}+\sum_{i=1}^{l} w_{i} f_{i, j}}{\sum_{i=1}^{d^{2}} h_{i}^{2} z_{i, j}^{2}} \leq 1, \quad j=1, \ldots, n . \\
& h_{i}^{2}, u_{i}, w_{i} \geq 0
\end{aligned}
$$

In the next step, the total efficiency value for $\mathrm{DMU}_{\mathrm{o}}$ can be expressed by considering the average efficiency as follows:

$$
\max \frac{1}{3}\left(\frac{\sum_{i=1}^{d^{1}} h_{i}^{1} z_{i, o}^{1}}{\sum_{i=1}^{m} v_{i} x_{i, o}}+\frac{\sum_{i=1}^{d^{2}} h_{i}^{2} z_{i, o}^{2}}{\sum_{i=1}^{d^{1}} h_{i}^{1} z_{i, o}^{1}+\sum_{i=1}^{l} w_{i} f_{i, o}}+\frac{\sum_{i=1}^{s} u_{i} y_{i, o}+\sum_{i=1}^{l} w_{i} f_{i, o}}{\sum_{i=1}^{d^{2}} h_{i}^{2} z_{i, o}^{2}}\right),
$$




$$
\begin{aligned}
& \text { subject to } \frac{\sum_{i=1}^{d^{1}} h_{i}^{1} z_{i, j}^{1}}{\sum_{i=1}^{m} v_{i} x_{i, j}} \leq 1, \\
& \frac{\sum_{i=1}^{d^{2}} h_{i}^{2} z_{i, j}^{2}}{\sum_{i=1}^{d^{1}} h_{i}^{1} z_{i, j}^{1}+\sum_{i=1}^{l} w_{i} f_{i, j}} \leq 1, \quad j=1, \ldots, n . \\
& \frac{\sum_{i=1}^{s} u_{i} y_{i, j}+\sum_{i=1}^{l} w_{i} f_{i, j}}{\sum_{i=1}^{d^{2}} h_{i}^{2} z_{i, j}^{2}} \leq 1, \\
& u_{i}, w_{i}, h_{i}^{1}, h_{i}^{2}, v_{i} \geq 0,
\end{aligned}
$$

Programing problem (4) is a nonlinear programming problem and regarding that solving nonlinear programming problems is difficult in terms of computational speed and accuracy in comparison with linear programming models, so, in the following, with the method having been described in the sources (Liang et al., 2011; Charnes \& Cooper, 1962), the problem is explained in Eq. (4). For linearization programming problem (4), we define the values of $\alpha, \beta, t_{i}(i=1, \ldots .4)$ as follows:

$$
t_{1}:=\frac{1}{\sum_{i=1}^{m} v_{i} x_{i, o}}, \quad t_{2}:=\frac{1}{\sum_{i=1}^{d^{1}} h_{i}^{1} z_{i, o}^{1}}, \quad t_{3}:=\frac{1}{\sum_{i=1}^{d^{2}} h_{i}^{2} z_{i, o}^{2}}, \quad t_{4}:=\frac{1}{\sum_{i=1}^{d^{1}} h_{i}^{1} z_{i, o}^{1}+\sum_{i=1}^{l} w_{i} f_{i, o}},
$$

and also:

$$
\alpha:=\frac{t_{4}}{t_{3}}, \quad \beta:=\frac{t_{1}}{t_{2}}
$$

Assuming the values mentioned above, by using the mathematical simplification operation, we can revise model (4) as follows:

$$
\begin{aligned}
& \max \frac{1}{3}\left(\beta+\alpha+\sum_{i=1}^{s} \hat{u}_{i} y_{i, o}+\sum_{i=1}^{l} \widehat{w}_{i} f_{i, o}\right), \\
& \text { subject to } \boldsymbol{\alpha} \sum_{i=1}^{d^{2}} \hat{h}_{i}^{2} z_{i, j}^{2}-\sum_{i=1}^{d^{1}} \hat{h}_{i}^{1} z_{i, j}^{1}-\sum_{i=1}^{l} \widehat{w}_{i} f_{i, j} \leq 0, \\
& \sum_{i=1}^{s} \hat{u}_{i} y_{i, j}+\sum_{i=1}^{l} \widehat{w}_{i} f_{i, j}-\sum_{i=1}^{d^{2}} \hat{h}_{i}^{2} z_{i, j}^{2} \leq 0, \\
& \sum_{i=1}^{d^{1}} \hat{h}_{i}^{1} z_{i, j}^{1}-\sum_{i=1}^{m} \hat{v}_{i} x_{i, j} \leq 0, \quad j=1, \ldots . n, \\
& \sum_{i=1}^{d^{1}} \hat{h}_{i}^{1} z_{i, 0}^{1}-\boldsymbol{\beta} \sum_{i=1}^{m} \hat{v}_{i} x_{i, 0}=0, \\
& \sum_{i=1}^{d^{2}} \hat{h}_{i}^{2} z_{i, o}^{2}=1, \\
& \alpha \sum_{i=1}^{l} \widehat{w}_{i} f_{i, o}+\sum_{i=1}^{d^{1}} \hat{h}_{i}^{1} z_{i, o}^{1}=1, \\
& \hat{u}_{i}, \widehat{w}_{i}, \hat{h}_{i}^{1}, \hat{h}_{i}^{2}, \hat{v}_{i} \geq 0, \quad \beta, \alpha \leq 1,
\end{aligned}
$$

In the above relations, the values $\hat{v}_{i}, \hat{h}_{i}^{1}, \hat{h}_{i}^{2}, \widehat{w}_{i} \cdot \hat{u}_{i}$ are defined as follows:

$$
\hat{u}_{i}=t_{3} u_{i}, \quad \widehat{w}_{i}=t_{3} w_{i}, \quad \hat{h}_{i}^{1}=t_{4} h_{i}^{1}, \quad \hat{h}_{i}^{2}=t_{3} h_{i}^{2}, \quad \hat{v}_{i}=t_{4} v_{i},
$$

The scheduling problem (5) is also a nonlinear programming problem, but if we consider the values of $\alpha, \beta$ as constant values, this model becomes a linear programming problem. In practice, in order to solve this problem, regarding that the values of $\alpha, \beta$ are always defined in the interval between zero and 
one, for given $\alpha$ and $\beta$, we select an initial point like $\alpha_{0}, \beta_{0}$, and then, we increase these values with the step lengths $\delta \alpha, \delta \beta$ in the form of the following relations to the unit value.

$$
\alpha_{i}:=\alpha_{0}+i \delta \alpha, \quad \beta_{i}:=\beta_{0}+i \delta \beta
$$

Then we calculate problem (5) for each of these values to find the optimal value of the model. Assuming that the values of $\alpha, \beta$ are constants, we can simplify the objective function of programming problem (5) as the following equation, and in practice, we use this objective function for the computation.

$$
\max \sum_{i=1}^{s} \widehat{u}_{i} y_{i, o}+\sum_{i=1}^{l} \widehat{w}_{i} f_{i, o},
$$

\section{Investigating the three-stage network data envelopment analysis model with two feedback}

In this section, we Fig. 2 which is the three-stage model with series structure that has two feedback between the third and the second stages, and the third and the first step.

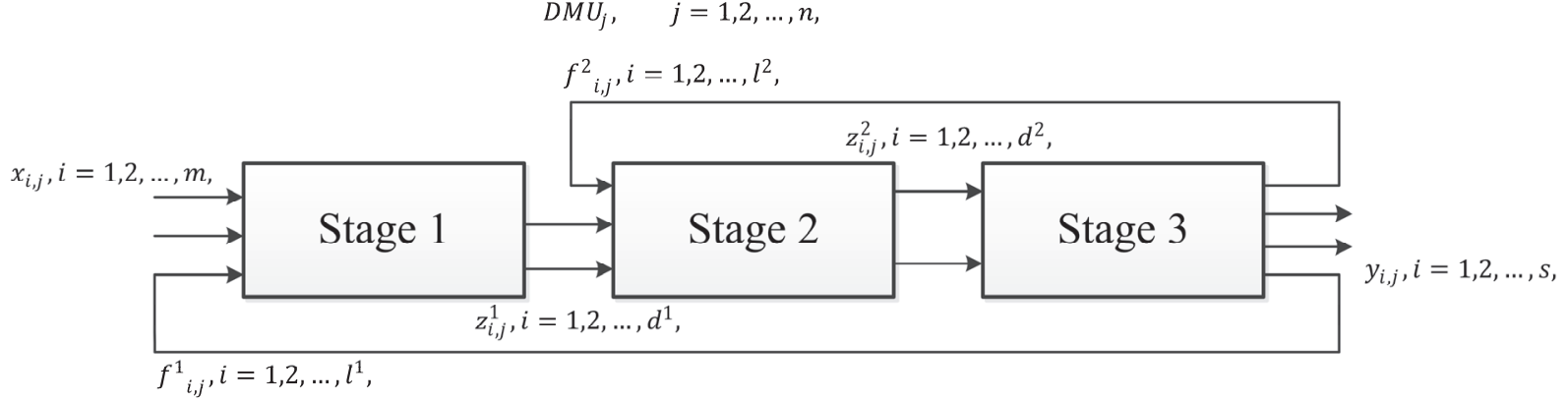

Fig. 2. Three stage model with two feedback

In the following, we examine the efficiency of each of the stages. According to Fig. 2, the efficiency of the first step for arbitrary DMU, such as $\mathrm{j}=\mathrm{o}$, can be calculated as follows:

$$
\begin{aligned}
& \max \frac{\sum_{i=1}^{d^{1}} h_{i}^{1} z_{i, o}^{1}}{\sum_{i=1}^{m} v_{i} x_{i, o+} \sum_{i=1}^{l^{1}} w_{i}^{1} f^{1}{ }_{i, o}}, \\
& \text { subject to } \quad \frac{\sum_{i=1}^{d^{1}} h_{i}^{1} z_{i, j}^{1}}{\sum_{i=1}^{m} v_{i} x_{i, j+} \sum_{i=1}^{l^{1}} w_{i}^{1} f^{1}{ }_{i, j}} \leq 1, \quad \mathrm{j}=1, \cdots, \mathrm{n} . \\
& h_{i}^{1}, v_{i}, w_{i}^{1} \geq 0
\end{aligned}
$$

To calculate the efficiency of the second stage we have:

$\max \frac{\sum_{i=1}^{d^{2}} h_{i}^{2} z_{i, o}^{2}}{\sum_{i=1}^{d^{1}} h_{i}^{1} z_{i, o}^{1}+\sum_{i=1}^{l^{2}} w_{i}^{2} f^{2}{ }_{i, o}}$

subject to $\frac{\sum_{i=1}^{d^{2}} h_{i}^{2} z_{i, j}^{2}}{\sum_{i=1}^{d^{1}} h_{i}^{1} z_{i, j}^{1}+\sum_{i=1}^{l^{2}} w_{i}^{2} f^{2}{ }_{i, j}} \leq 1, \quad j=1, \ldots, n$.

$h_{i}^{1}, h_{i}^{2}, w_{i}^{2} \geq 0$

The efficiency of the third stage is calculated as follows:

$$
\begin{aligned}
& \max \frac{\sum_{i=1}^{S} u_{i} y_{i, o}+\sum_{i=1}^{l^{1}} w_{i}^{1} f^{1}{ }_{i, o}+\sum_{i=1}^{l^{2}} w_{i}^{2} f^{2}{ }_{i, o}}{\sum_{i=1}^{d^{2}} h_{i}^{2} z_{i, o}^{2}} \\
& \text { subject to } \frac{\sum_{i=1}^{S} u_{i} y_{i, j}+\sum_{i=1}^{l^{1}} w_{i}^{1} f^{1}{ }_{i, j}+\sum_{i=1}^{l^{2}} w_{i}^{2} f^{2}{ }_{i, j}}{\sum_{i=1}^{d^{2}} h_{i}^{2} z_{i, j}^{2}} \leq 1, \quad j=1, \ldots, n . \\
& h_{i}^{2}, u_{i}, w_{i}^{1}, w_{i}^{2} \geq 0
\end{aligned}
$$


The total efficiency for $\mathrm{DMU}_{\mathrm{j}}$ with the value of $\mathrm{j}=\mathrm{o}$ ( $\mathrm{o}$ is an arbitrary value and $1 \leq \mathrm{o} \leq \mathrm{n}$ ) can be expressed by considering the average efficiency as follows:

$$
\begin{aligned}
& \max \frac{1}{3}\left(\frac{\sum_{i=1}^{d^{1}} h_{i}^{1} z_{i, o}^{1}}{\sum_{i=1}^{m} v_{i} x_{i, o+} \sum_{i=1}^{l^{1}} w_{i}^{1} f^{1}{ }_{i, o}}+\frac{\sum_{i=1}^{d^{2}} h_{i}^{2} z_{i, o}^{2}}{\sum_{i=1}^{d^{1}} h_{i}^{1} z_{i, o}^{1}+\sum_{i=1}^{l^{2}} w_{i}^{2} f^{2}{ }_{i, o}}\right. \\
& \left.+\frac{\sum_{i=1}^{S} u_{i} y_{i, o}+\sum_{i=1}^{l^{1}} w_{i}^{1} f^{1}{ }_{i, o}+\sum_{i=1}^{l^{2}} w_{i}^{2} f^{2}{ }_{i, o}}{\sum_{i=1}^{d^{2}} h_{i}^{2} z_{i, o}^{2}}\right) \\
& \text { subject to } \frac{\sum_{i=1}^{d^{1}} h_{i}^{1} z_{i, j}^{1}}{\sum_{i=1}^{m} v_{i} x_{i, j+} \sum_{i=1}^{l^{1}} w_{i}^{1} f^{1}{ }_{i, j}} \leq 1 \text {, } \\
& \frac{\sum_{i=1}^{d^{2}} h_{i}^{2} z_{i, j}^{2}}{\sum_{i=1}^{d^{1}} h_{i}^{1} z_{i, j}^{1}+\sum_{i=1}^{l} w_{i} f^{2}{ }_{i, j}} \leq 1, \mathrm{j}=1, \ldots . \mathrm{n}, \\
& \frac{\sum_{i=1}^{S} u_{i} y_{i, j}+\sum_{i=1}^{l^{1}} w_{i}^{1} f^{1}{ }_{i, j}+\sum_{i=1}^{l^{2}} w_{i}^{2} f^{2}{ }_{i, j}}{\sum_{i=1}^{d^{2}} h_{i}^{2} z_{i, j}^{2}} \leq 1, \\
& u_{i}, w_{i}, h_{i}^{1}, h_{i}^{2}, v_{i} \geq 0 \text {, }
\end{aligned}
$$

Problem (9) is a nonlinear programming problem, but it can be transformed into a linear programming problem, as discussed in the previous section. For this purpose, the values of $\alpha, \beta, t_{i}(i=1, \ldots .4)$ are defined as follows:

$$
\begin{gathered}
t_{1}:=\frac{1}{\sum_{i=1}^{d^{1}} h_{i}^{1} z_{i, o}^{1}}, \quad t_{2}:=\frac{1}{\sum_{i=1}^{m} v_{i} x_{i, o} \sum_{i=1}^{l^{1}} w_{i}^{1} f^{1}{ }_{i, o}}, \\
t_{3}:=\frac{1}{\sum_{i=1}^{d^{2}} h_{i}^{2} z_{i, o}^{2}}, \quad t_{4}:=\frac{1}{\sum_{i=1}^{d^{1}} h_{i}^{1} z_{i, o}^{1}+\sum_{i=1}^{l^{2}} w_{i}^{2} f^{2}{ }_{i, o}}, \\
\beta:=\frac{t_{2}}{t_{1}}, \quad \alpha:=\frac{t_{4}}{t_{3}}
\end{gathered}
$$

Now, assuming the above values, we can specify nonlinear programming problem (9) as follows:

$$
\begin{aligned}
& \max \frac{1}{3}\left(\beta+\alpha+\sum_{i=1}^{s} \hat{u}_{i} y_{i, o}+\sum_{i=1}^{l^{1}} \widehat{w}_{i}{ }_{i} f^{1}{ }_{i, o}+\sum_{i=1}^{l^{2}} \widehat{w}_{i}{ }_{i} f^{2}{ }_{i, o}\right) \\
& \text { subject to } \sum_{i=1}^{d^{1}} \hat{h}_{i}^{1} z_{i, j}^{1}-\sum_{i=1}^{m} \hat{v}_{i} x_{i, j}-\sum_{i=1}^{l^{1}} \widehat{w}_{i}^{1} f^{1}{ }_{i, j} \leq 0, \\
& \sum_{i=1}^{d^{2}} \hat{h}_{i}^{2} z_{i, j}^{2}-\sum_{i=1}^{d^{1}} \hat{h}_{i}^{1} z_{i, j}^{1}-\sum_{i=1}^{l^{2}} \widehat{w}_{i}{ }^{2} f^{2}{ }_{i, j} \leq 0, \\
& \sum_{i=1}^{s} \hat{u}_{i} y_{i, j}+\sum_{i=1}^{l^{1}} \widehat{w}_{i}{ }^{1} f^{1}{ }_{i, j}+\sum_{i=1}^{l^{2}} \widehat{w}_{i}{ }^{2} f^{2}{ }_{i, j}-\sum_{i=1}^{d^{2}} \hat{h}_{i}^{2} z_{i, j}^{2} \leq 0, \mathrm{j}=1, \ldots \mathrm{n} \\
& \sum_{i=1}^{d^{1}} \hat{h}_{i}^{1} z_{i, 0}^{1}-\boldsymbol{\beta}\left(\sum_{i=1}^{m} \hat{v}_{i} x_{i, 0}+\sum_{i=1}^{l^{1}} \widehat{w}_{i}{ }^{1} f^{1}{ }_{i, j}\right)=0, \\
& \sum_{i=1}^{d^{2}} \hat{h}_{i}^{2} z_{i, o}^{2}=1, \\
& \alpha\left(\sum_{i=1}^{l^{2}} \widehat{w}_{i}{ }^{2} f^{2}{ }_{i, j}+\sum_{i=1} \hat{h}_{i}^{1} z_{i, o}^{1}\right)=1, \\
& \hat{u}_{i}, \widehat{w}_{i}{ }^{1}, \widehat{w}_{i}{ }^{2}, \hat{h}_{i}^{1}, \hat{h}_{i}^{2}, \hat{v}_{i} \geq 0,0 \leq \beta, \alpha \leq 1,
\end{aligned}
$$


For the above relationships, the values of $\hat{v}_{i}, \hat{h}_{i}^{1}, \hat{h}_{i}^{2}, \widehat{w}_{i}^{1}, \widehat{w}_{i}^{2} \cdot \widehat{u}_{i}$ are defined as follows:

$$
\hat{u}_{i}=t_{3} u_{i}, \quad \widehat{w}_{i}^{1}=t_{3} w_{i}^{1}, \quad \widehat{w}_{i}^{2}=t_{3} w_{i}^{2}, \quad \hat{h}_{i}^{1}=t_{3} h_{i}^{1}, \quad \hat{h}_{i}^{2}=t_{3} h_{i}^{2}, \quad \hat{v}_{i}=t_{3} v_{i},
$$

Programming problem (10) is a nonlinear programming problem, which, like the discussion of Section 2 , by assuming constant numbers for $\alpha, \beta$, this problem becomes a linear programming problem, and, as discussed in Section 2, by choosing the initial values for $\alpha, \beta$ and step-by-step increments up to the unit value, this problem is solved and we find the optimal value for this problem. In problem (10), if $\alpha$, $\beta$ are considered as constants, then the objective function of programming problem (10) can be considered as follows:

$\max \sum_{i=1}^{s} \hat{u}_{i} y_{i, o}+\sum_{i=1}^{l^{1}} \widehat{w}^{1}{ }_{i} f^{1}{ }_{i, o}+\sum_{i=1}^{l^{2}} \widehat{w}_{i}{ }_{i} f^{2}{ }_{i, o}$,

\section{Two-stage model for examining the efficiency of the stages}

In the discussion of Sections 2 and 3, the efficiency of the overall system and the efficiency of each stage, regardless of the relation to other stages, were examined, but sometimes one of the stages of these models has importance and we need to examine the efficiency of this stage. On the other hand, due to the association of the stages with each other, the efficiency for these stages cannot be calculated separately, so we use a two- stage programming model to examine the efficiency of each stage.

\subsection{Two-stage model for a three-stage model with a feedback}

For the model of Fig. 2, if the goal is to increase the efficiency level of the first stage, then the efficiency of the first stage has the most importance and the efficiency of the second and third stages will depend on the efficiency of the first stage. In this case, we consider the two-stage nonlinear programming problem below.

$$
\begin{aligned}
& \text { (p1): } \theta^{1}:=\max \frac{\sum_{i=1}^{d^{1}} h_{i}^{1} z_{i, o}^{1}}{\sum_{i=1}^{m} v_{i} x_{i, o}}, \\
& \text { (p2): } \quad \theta^{2,3}:=\max \frac{1}{2}\left(\frac{\sum_{i=1}^{d^{2}} h_{i}^{2} z_{i, o}^{2}}{\sum_{i=1}^{d^{1}} h_{i}^{1} z_{i, j}^{1}+\sum_{i=1}^{l} w_{i} f_{i, j}}+\frac{\sum_{i=1}^{s} u_{i} y_{i, o}+\sum_{i=1}^{l} w_{i} f_{i, o}}{\sum_{i=1}^{d^{2}} h_{i}^{2} z_{i, o}^{2}}\right), \\
& \text { subject to } \frac{\sum_{i=1}^{d^{1}} h_{i}^{1} z_{i, o}^{1}}{\sum_{i=1}^{m} v_{i} x_{i, o}} \leq 1 \text {, } \\
& \frac{\sum_{i=1}^{d^{2}} h_{i}^{2} z_{i, o}^{2}}{\sum_{i=1}^{d^{1}} h_{i}^{1} z_{i, j}^{1}+\sum_{i=1}^{l} w_{i} f_{i, j}} \leq 1, \\
& \frac{\sum_{i=1}^{s} u_{i} y_{i, o}+\sum_{i=1}^{l} w_{i} f_{i, o}}{\sum_{i=1}^{d^{2}} h_{i}^{2} z_{i, o}^{2}} \leq 1, \\
& u_{i}, w_{i}, h_{i}^{1}, h_{i}^{2}, v_{i} \geq 0 \text {, }
\end{aligned}
$$

Two-stage programming problem (11) is a nonlinear problem that can be transformed into a two-stage linear programming problem by using the methods discussed in Section 2. For this purpose, by considering the terms defined in Section 2, we have the following programming problem:

$$
\begin{aligned}
& (p 1): \theta^{1}:=\max \beta, \\
& (p 2): \quad \theta^{2,3}:=\max \frac{1}{2}\left(\alpha+\sum_{i=1}^{s} \hat{u}_{i} y_{i, o}+\sum_{i=1}^{l^{1}} \widehat{w}^{1}{ }_{i} f^{1}{ }_{i, o}\right), \\
& \text { subject to } \alpha \sum_{i=1}^{d^{2}} \hat{h}_{i}^{2} z_{i, j}^{2}-\sum_{i=1}^{d^{1}} \hat{h}_{i}^{1} z_{i, j}^{1}-\sum_{i=1}^{l} \widehat{w}_{i} f_{i, j} \leq 0,
\end{aligned}
$$




$$
\begin{aligned}
& \sum_{i=1}^{s} \hat{u}_{i} y_{i, j}+\sum_{i=1}^{l} \widehat{w}_{i} f_{i, j}-\sum_{i=1}^{d^{2}} \hat{h}_{i}^{2} z_{i, j}^{2} \leq 0, \\
& \sum_{i=1}^{d^{1}} \hat{h}_{i}^{1} z_{i, j}^{1}-\sum_{i=1}^{m} \hat{v}_{i} x_{i, j} \leq 0, j=1, \ldots . n \text {, } \\
& \sum_{i=1}^{d^{1}} \hat{h}_{i}^{1} z_{i, 0}^{1}-\beta \sum_{i=1}^{m} \hat{v}_{i} x_{i, 0}=0, \\
& \sum_{i=1}^{d^{2}} \hat{h}_{i}^{2} z_{i, o}^{2}=1 \\
& \alpha \sum_{i=1}^{l} \widehat{w}_{i} f_{i, o}+\sum_{i=1}^{d^{1}} \hat{h}_{i}^{1} z_{i, o}^{1}=1, \\
& \widehat{u}_{i}, \widehat{w}_{i}, \hat{h}_{i}^{1}, \hat{h}_{i}^{2}, \hat{v}_{i} \geq 0,0 \leq \beta, \alpha \leq 1 \text {, }
\end{aligned}
$$

For the problem mentioned above, the first goal is to maximize $\theta^{1}$ and the next goal is to maximize $\theta^{2,3}$. Similarly, with the above discussion, in the case where the efficiency of the second stage has more importance than the other two stages and the goal is to maximize the efficiency of the second stage, then the two-stage programming problem with objective function mentioned below and the restrictions of programming problem (11) are modeled.

$$
\begin{aligned}
& (p 1): \theta^{2}:=\max \alpha, \\
& (p 2): \theta^{1,3}:=\max \frac{1}{2}\left(\beta+\sum_{i=1}^{s} \hat{u}_{i} y_{i, o}+\sum_{i=1}^{l^{1}} \widehat{w}^{1}{ }_{i} f^{1}{ }_{i, o}\right)
\end{aligned}
$$

Also, in the case that the efficiency of the third stage has importance, the two-stage programming problem is modeled by the following objective function and the restrictions of two-stage programming problem (12):

$$
\begin{aligned}
& (p 1): \theta^{3}:=\max \sum_{i=1}^{s} \hat{u}_{i} y_{i, o}+\sum_{i=1}^{l^{1}} \widehat{w}^{1}{ }_{i} f^{1}{ }_{i, o}, \\
& (p 2): \theta^{1,2}:=\max \frac{1}{2}(\beta+\alpha),
\end{aligned}
$$

The numerical solution method for the two-stage programming problems presented in this section will be discussed in sub-section 3.4.

\subsection{Two-stage model for three-stage system with two feedback}

In the review of the efficiency of the three-stage model with two feedback (Fig. 2), in the cases where the efficiency of each stage has more importance, as discussed in the three-stage model with a feedback, the following discussion can be presented. If the first goal is to maximize the efficiency of the first stage and then maximize the efficiency of the second and third stages, in this problem, we have the two-stage programming below:

$$
\begin{aligned}
& (p 1): \theta^{1}:=\max \frac{\sum_{i=1}^{d^{1}} h_{i}^{1} z_{i, o}^{1}}{\sum_{i=1}^{m} v_{i} x_{i, o+} \sum_{i=1}^{l^{1}} w_{i}^{1} f^{1}{ }_{i, o}}, \\
& (p 2): \quad \theta^{2,3}:=\max \frac{1}{2}\left(\frac{\sum_{i=1}^{d^{2}} h_{i}^{2} z_{i, o}^{2}}{\sum_{i=1}^{d^{1}} h_{i}^{1} z_{i, o}^{1}+\sum_{i=1}^{l^{2}} w_{i}^{2} f^{2}{ }_{i, o}}+\frac{\sum_{i=1}^{s} u_{i} y_{i, o}+\sum_{i=1}^{l^{1}} w_{i}^{1} f^{1}{ }_{i, o}+\sum_{i=1}^{l^{2}} w_{i}^{2} f^{2}{ }_{i, o}}{\sum_{i=1}^{d^{2}} h_{i}^{2} z_{i, o}^{2}}\right)
\end{aligned}
$$




$$
\begin{aligned}
& \text { subject to } \frac{\sum_{i=1}^{d^{1}} h_{i}^{1} z_{i, j}^{1}}{\sum_{i=1}^{m} v_{i} x_{i, j} \sum_{i=1}^{l^{1}} w_{i}^{1} f^{1}{ }_{i, j}} \leq 1, \\
& \frac{\sum_{i=1}^{d^{2}} h_{i}^{2} z_{i, j}^{2}}{\sum_{i=1}^{d^{1}} h_{i}^{1} z_{i, j}^{1}+\sum_{i=1}^{l^{2}} w_{i}^{2} f^{2}{ }_{i, j}} \leq 1, \mathrm{j}=1, \ldots \mathrm{n}, \\
& \frac{\sum_{i=1}^{S} u_{i} y_{i, j}+\sum_{i=1}^{l^{1}} w_{i}^{1} f^{1}{ }_{i, j}+\sum_{i=1}^{l^{2}} w_{i}^{2} f^{2}{ }_{i, j}}{\sum_{i=1}^{d^{2}} h_{i}^{2} z_{i, j}^{2}} \leq 1, \\
& u_{i}, w_{i}, h_{i}^{1}, h_{i}^{2}, v_{i} \geq 0 .
\end{aligned}
$$

Given that programming problem (15) is a nonlinear programming problem, considering the terms defined in Section 3, this problem is transformed into the following two-stage programming model; the following problem can be transformed into a two-stage linear programming problem by considering the values of this problem to be constant.

$(p 1): \theta^{1}:=\max \beta$,

(p2): $\theta^{2,3}:=\max \frac{1}{2}\left(\alpha+\sum_{i=1}^{s} \hat{u}_{i} y_{i, o}+\sum_{i=1}^{l^{1}} \widehat{w}^{1}{ }_{i} f^{1}{ }_{i, o}+\sum_{i=1}^{l^{2}} \widehat{w}^{2}{ }_{i} f^{2}{ }_{i, o}\right)$

subject to

$\sum_{i=1}^{d^{1}} \hat{h}_{i}^{1} z_{i, j}^{1}-\sum_{i=1}^{m} v_{i} x_{i, j-} \sum_{i=1}^{l^{1}} w_{i}^{1} f^{1}{ }_{i, j} \leq 0$

$\sum_{i=1}^{d^{2}} \hat{h}_{i}^{2} z_{i, j}^{2}-\sum_{i=1}^{d^{1}} \hat{h}_{i}^{1} z_{i, j}^{1}-\sum_{i=1}^{l^{2}} \widehat{w}_{i}^{2} f^{2}{ }_{i, j} \leq 0$,

$\sum_{i=1}^{s} \hat{u}_{i} y_{i, j}+\sum_{i=1}^{l^{1}} \widehat{w}_{i}{ }^{1} f^{1}{ }_{i, j}+\sum_{i=1}^{l^{2}} \widehat{w}_{i}{ }^{2} f^{2}{ }_{i, j}-\sum_{i=1}^{d^{2}} \hat{h}_{i}^{2} z_{i, j}^{2} \leq 0, \mathrm{j}=1, \ldots \mathrm{n}$

$\sum_{i=1}^{d^{1}} \hat{h}_{i}^{1} z_{i, 0}^{1}-\boldsymbol{\beta}\left(\sum_{i=1}^{m} \hat{v}_{i} x_{i, 0}+\sum_{i=1}^{l^{1}} \widehat{w}_{i}{ }^{1} f^{1}{ }_{i, j}\right)=0$,

$\sum_{i=1}^{d^{2}} \hat{h}_{i}^{2} z_{i, o}^{2}=1$

$\alpha\left(\sum_{i=1}^{l^{2}} \widehat{w}_{i}^{2} f^{2}{ }_{i, j}+\sum_{i=1}^{d^{1}} \hat{h}_{i}^{1} z_{i, o}^{1}\right)=1$,

$\hat{u}_{i}, \widehat{w}_{i}{ }^{1}, \widehat{w}_{i}{ }^{2}, \hat{h}_{i}^{1}, \hat{h}_{i}^{2}, \hat{v}_{i} \geq 0,0 \leq \beta, \alpha \leq 1$.

Also, in cases where maximizing the second stage has more importance than the first and second stages, then the two-stage programming problem can be expressed with problem restrictions (16) and the following objective function:

$(p 1): \theta^{2}:=\max \alpha$,

(p2): $\quad \theta^{1,3}:=\max \frac{1}{2}\left(\beta+\sum_{i=1}^{s} \hat{u}_{i} y_{i, o}+\sum_{i=1}^{l^{1}} \widehat{w}^{1}{ }_{i} f^{1}{ }_{i, o}+\sum_{i=1}^{l^{2}} \widehat{w}_{i}{ }_{i} f^{2}{ }_{i, o}\right)$. 
In the case that maximizing the efficiency of the second stage has more importance than the other two stages, the problem is considered as a two-stage programming problem with the restrictions of twostage programming problem (16) and the following objective function:

$$
\begin{aligned}
& (p 1): \theta^{3}:=\max \sum_{i=1}^{s} \hat{u}_{i} y_{i, o}+\sum_{i=1}^{l^{1}} \widehat{w}_{i}{ }_{i} f^{1}{ }_{i, o}+\sum_{i=1}^{l^{2}} \widehat{w}^{2}{ }_{i} f^{2}{ }_{i, o}, \\
& \text { (p2): } \quad \theta^{1,2}:=\max \frac{1}{2}(\alpha+\beta) .
\end{aligned}
$$

For the above problem, the first goal is to maximize $\theta^{3}$ and the next goal is to maximize $\theta^{1,2}$.

\subsection{Providing a linearization-based method for solving the two-stage programming problem}

The two-stage programming problems raised in sub-sections 4.1 and 4.2 have a nonlinear structure, but if we consider $\alpha, \beta$ as constant numbers in these problems, then these problems are transformed into a two-stage linear programming problem, and solving this linear problem will be simpler in comparison with the nonlinear mode in terms of computational volume. Solving the two-stage linear programming problem has been studied in the various papers, (See Sakawa \& Nishizaki, 2002; Bialas \& Karvan, 1984; Candler \& Townsley, 1982; Xie et al., 2017; Ahlatcioglu \& Tiryaki, 2007; Sakawa and Nishizaki, 2001). It is possible to solve the two-stage programming problems by using the methods mentioned in the resources above. However, a more appropriate method can be proposed compared with the methods presented in these papers based on the principles of the linearization proposed in the previous sections in order to solve the two-stage problems. To solve all of the preceding two-stage problems, first, the values of $\beta$ and $\alpha$ are assumed to be constant values, as discussed in the previous sections. With this assumption, programming problem (p2) in the two-stage problems is equivalent to solving the problems proposed in sections 2 and 3. We describe solving method of linear programming problem (16). Since the process is similar for other problems, and we do not repeat them again. First, we select the length value of stage $\delta \beta$ and then we calculate the value of $\beta_{i}=\beta_{0}+i \delta \beta$ with the initial point $\beta_{0}$ for $\mathrm{i}=$ $1,2,3, \ldots$ and in the next step, for each $\beta_{i}$ assuming the length of the step $\delta \alpha$, we consider the values of

$\alpha_{i}=\alpha_{0}+i \delta \alpha$ for $i=1,2,3, \ldots$. Then, by using these values in two-stage programming problem (16), we solve problem (p2) for each $\beta_{i}$ and $\alpha_{\mathrm{i}}$, and then we select the maximum value of $\beta$ that problem (p2) is feasible. After choosing appropriate $\beta$, in the next section we select the maximum values for $\alpha$ and $\hat{u}_{i}, \widehat{w}^{1}{ }_{i}, \widehat{w}^{2}{ }_{i}$, in a way that the following expression has the highest value.

$$
\frac{1}{2}\left(\alpha+\sum_{i=1}^{s} \hat{u}_{i} y_{i, o}+\sum_{i=1}^{l^{1}} \widehat{w}_{i}^{1} f^{1}{ }_{i, o}+\sum_{i=1}^{l^{2}} \widehat{w}_{i}^{2} f^{2}{ }_{i, o}\right) .
$$

In this case, the resulted values represent the answer of the two-stage programming problem. In the following, in order to examine the theoretical discussion, we will present the numerical results in the next section.

\section{Examining numerical results}

In this section, we check a few numerical examples for the contents of the preceding sections and examine the obtained results in the form of the chart and table. It should be noted that the numerical results have been programmed by using Wolfram Mathematica 9.0 and Matlab 2014.b software. It should be noted that in all numerical results, the initial values $\alpha_{0}=\beta_{0}=0$ and the value of $\delta \alpha=\delta \beta=$ 0.1 are considered as the stage length, although it is possible by selecting smaller values for this stage length to increase the decimal accuracy of the results. The numerical values for the input and output parameters for three DMUs have been given in Table 1. By using the values in this table, we will 
examine the three-stage data envelopment analysis model with one feedback, namely, models (1) to (6). In Table 2, for the models (1) to (4), the numerical results without linearization have been presented by using the FMINCON internal function of MATLAB software and applying the interior-point default algorithm. Also, the results of linearization for these models by using the proposed method have been presented in Table 3. By comparing the obtained results, it is seen that the proposed linear approximation in comparison with the nonlinear solving, has a proper accuracy, and if the length of the stages increases, the accuracy of the results of the linearization will be closer to the actual answer.

\section{Table 1}

Numerical data for two-stage model for three-stage system with one feedback

\begin{tabular}{|c|c|c|c|c|c|c|c|c|c|c|c|c|c|c|c|}
\hline DMU & $\mathbf{x}_{1, j}$ & $\mathbf{x}_{2, \mathbf{j}}$ & $\mathbf{x}_{3, \mathbf{j}}$ & $\mathbf{x}_{4, \mathbf{j}}$ & $\mathbf{x}_{5, j}$ & $\mathrm{z}_{1, \mathrm{j}}^{1}$ & $\mathbf{z}_{2, \mathrm{j}}^{1}$ & $\mathrm{z}_{1, \mathrm{j}}^{2}$ & $\mathrm{z}_{2, \mathrm{j}}^{2}$ & $\mathrm{z}_{3, \mathrm{j}}^{2}$ & $\mathbf{f}_{1, j}$ & $\mathbf{f}_{2, j}$ & $\mathbf{y}_{1, j}$ & $\mathbf{y}_{2, \mathbf{j}}$ & $y_{3, j}$ \\
\hline 1 & 50 & 43 & 25 & 32 & 45 & 27 & 20 & 63 & 10 & 44 & 10 & 11 & 17 & 16 & 15 \\
\hline 2 & 32 & 52 & 27 & 20 & 40 & 30 & 22 & 10 & 10 & 10 & 40 & 30 & 72 & 50 & 80 \\
\hline 3 & 30 & 50 & 32 & 50 & 50 & 71 & 55 & 43 & 71 & 49 & 15 & 14 & 57 & 80 & 14 \\
\hline
\end{tabular}

Table 2

Numerical results for nonlinear models (1) to (4), without linearization for data in Table 1

\begin{tabular}{lllll}
\hline DMU & Overall efficiency of model (4) & Stage 1 efficiency & Stage 2 efficiency & Stage 3 efficiency \\
\hline $\mathbf{1}$ & 0.8209 & 0.8900 & 1.0000 & 1.0000 \\
$\mathbf{2}$ & 1.0000 & 1.0000 & 1.0000 & 1.0000 \\
$\mathbf{3}$ & 1.0000 & 1.0000 & 1.0000 & 1.0000 \\
\hline
\end{tabular}

Table 3

Numerical results for the data in Table 1 with values of $\delta \alpha=\delta \beta=0.1$

\begin{tabular}{llccccccc}
\hline DMU & $\begin{array}{l}\text { Overall efficiency of } \\
\text { model (4) }\end{array}$ & $\boldsymbol{\alpha}^{*}$ & $\boldsymbol{\beta}^{*}$ & Largest $\boldsymbol{\alpha}$ & Largest $\boldsymbol{\beta}$ & $\begin{array}{l}\text { Stage 1 } \\
\text { efficiency }\end{array}$ & $\begin{array}{l}\text { Stage 2 } \\
\text { efficiency }\end{array}$ & $\begin{array}{l}\text { Stage 3 } \\
\text { efficiency }\end{array}$ \\
\hline $\mathbf{1}$ & 0.8227 & 0.9000 & 0.7000 & 1.0000 & 0.8000 & 0.8000 & 1.0000 & 1.0000 \\
$\mathbf{2}$ & 1.0000 & 1.0000 & 1.0000 & 1.0000 & 1.0000 & 1.0000 & 1.0000 & 1.0000 \\
$\mathbf{3}$ & 1.0000 & 1.0000 & 0.4000 & 1.0000 & 1.0000 & 1.0000 & 1.0000 & 1.0000 \\
\hline
\end{tabular}

Also for the numerical results in Table 1, the results of the two-stage programming (12) - (14) have been presented in Table 3. Fig. 3 shows the Cantor chart and the three-dimensional chart of the total efficiency for the different values of $\alpha$ and $\beta$ for the first DMU. In the mentioned chart, $\theta^{\alpha}$ represents the total efficiency.

Table 4

Numerical results for two-stage problems of model 1 with values of $\delta \alpha=\delta \beta=0.1$

\begin{tabular}{llllllllll}
\hline \multicolumn{2}{l}{ Model (12) } & & & Model (13) & & Model (14) \\
DMU & $\begin{array}{l}\text { Stage 1 } \\
\text { efficiency }\end{array}$ & $\begin{array}{l}\text { Stage 2 } \\
\text { efficiency }\end{array}$ & $\begin{array}{l}\text { Stage 3 } \\
\text { efficiency }\end{array}$ & $\begin{array}{l}\text { Stage 1 } \\
\text { efficiency }\end{array}$ & $\begin{array}{l}\text { Stage 2 } \\
\text { efficiency }\end{array}$ & $\begin{array}{l}\text { Stage 3 } \\
\text { efficiency }\end{array}$ & $\begin{array}{l}\text { Stage 1 } \\
\text { efficiency }\end{array}$ & $\begin{array}{l}\text { Stage 2 } \\
\text { efficiency }\end{array}$ & $\begin{array}{l}\text { Stage 3 } \\
\text { efficiency }\end{array}$ \\
\hline 1 & 0.8000 & 0.8000 & 0.8529 & 0.6000 & 1.0000 & 0.8444 & 0.7000 & 1.0000 & $\mathbf{1 . 0 0 0 0}$ \\
2 & 1.0000 & 1.0000 & 1.0000 & 1.0000 & 1.0000 & 1.0000 & 1.0000 & 1.0000 & $\mathbf{1 . 0 0 0 0}$ \\
3 & 1.0000 & 1.0000 & 1.0000 & 1.0000 & 1.0000 & 1.0000 & 1.0000 & 1.0000 & $\mathbf{1 . 0 0 0 0}$ \\
\hline
\end{tabular}

In order to investigate the problems of three-stage models with series structure, we consider three DMUs with the data in Table 5.

Table 5

Numerical data for a two-stage model for three-stage system with two feedback

\begin{tabular}{|c|c|c|c|c|c|c|c|c|c|c|c|c|c|c|c|c|c|c|}
\hline DMU & $x_{1, j}$ & $\mathbf{x}_{2, \mathrm{j}}$ & $x_{3, j}$ & $x_{4, j}$ & $\mathbf{z}_{1, j}^{2}$ & $\mathrm{z}_{1, \mathrm{j}}^{1}$ & $\mathrm{z}_{2, \mathrm{j}}^{1}$ & $z_{1, j}^{2}$ & $\mathbf{z}_{2, \mathrm{j}}^{2}$ & $z_{3, j}^{2}$ & $\mathbf{f}_{1, j}^{1}$ & $\mathbf{f}_{2, \mathrm{j}}^{1}$ & $f_{2, j}^{2}$ & $f_{2, j}^{2}$ & $f_{3, j}^{2}$ & $\mathbf{y}_{1, j}$ & $\mathbf{y}_{2, \mathrm{j}}$ & $y_{3, j}$ \\
\hline 1 & 60 & 43 & 25 & 40 & 45 & 50 & 75 & 10 & 5 & 10 & 10 & 11 & 50 & 50 & 12 & 17 & 16 & 12 \\
\hline 2 & 32 & 52 & 27 & 20 & 40 & 17 & 12 & 53 & 15 & 44 & 18 & 38 & 20 & 20 & 15 & 14 & 50 & 50 \\
\hline 3 & 30 & 50 & 32 & 50 & 50 & 10 & 30 & 43 & 15 & 49 & 15 & 14 & 14 & 14 & 50 & 80 & 80 & 50 \\
\hline
\end{tabular}

In Table 6, the numerical results are obtained from models (6) to (9), without the linearization process by using the internal FMINCON function of the MATLAB software by applying the default interior- 
point algorithm. Also, the results of the proposed linearization for these models are shown in Table 6 . By comparing the obtained results, it is seen that the proposed linearization approximate, in comparison with nonlinear solving, has a proper accuracy and if the length of the stages increases, the accuracy of the results of the linearization will be closer to the actual answer.

Table 6

Numerical results for nonlinear models (6) to (9), without linearization for data in Table 5

\begin{tabular}{lllll}
\hline DMU & Overall efficiency of model (4) & Stage 1 efficiency & Stage 2 efficiency & Stage 3 efficiency \\
\hline $\mathbf{1}$ & 0.7161 & 1.0000 & 0.4166 & 1.0000 \\
$\mathbf{2}$ & 0.8491 & 0.2266 & 1.0000 & 1.0000 \\
$\mathbf{3}$ & 0.8596 & 0.8000 & 1.0000 & 1.0000 \\
\hline
\end{tabular}

Table 7

Numerical results for data in Table 5 with values of $\delta \alpha=\delta \beta=0.1$

\begin{tabular}{llllllllll}
\hline DMU & Overall efficiency of model (4) & \multirow{2}{*}{$\boldsymbol{\alpha}^{*}$} & $\boldsymbol{\beta}^{*}$ & $\begin{array}{l}\text { Largest } \\
\boldsymbol{\alpha}\end{array}$ & $\begin{array}{l}\text { Largest } \\
\boldsymbol{\beta}\end{array}$ & $\begin{array}{l}\text { Stage } \\
\text { efficiency }\end{array}$ & $\begin{array}{l}\text { Stage } \\
\text { efficiency }\end{array}$ & $\begin{array}{l}\text { Stage } \\
\text { efficiency }\end{array}$ \\
\hline 1 & 0.7100 & 0.1000 & 1.0000 & 0.4000 & 0.8000 & 1.0000 & 0.4000 & 1.0000 \\
2 & 0.8100 & 0.1000 & 0.2000 & 1.0000 & 0.4000 & 0.2000 & 0.1000 & 1.0000 \\
3 & 0.8300 & 0.9000 & 0.5000 & 1.0000 & 1.0000 & 0.8000 & 1.0000 & 1.0000 \\
\hline
\end{tabular}

Also, in order to examine the two-stage model proposed in Table 8, we consider four DMUs. The obtained results for the two-stage problems are presented in Table 9. The graphs for optimal values for the fourth DMU are plotted in Fig. 3. Also, for this mode, the optimal value is plotted in the Cantor graph and the total efficiency values have been represented by $\theta^{a}$.

\section{Table 8}

Numerical data for model 2

\begin{tabular}{lllllllllllllllll}
\hline $\mathbf{D M U}$ & $\mathbf{x}_{\mathbf{1}, \mathbf{j}}$ & $\mathbf{x}_{\mathbf{2} \mathbf{j}}$ & $\mathbf{x}_{\mathbf{3} \mathbf{j}}$ & $\mathbf{x}_{\mathbf{4}, \mathbf{j}}$ & $\mathbf{z}_{\mathbf{1}, \mathbf{j}}^{\mathbf{1}}$ & $\mathbf{z}_{\mathbf{2} \mathbf{j}}^{\mathbf{1}}$ & $\mathbf{z}_{\mathbf{1} \mathbf{j}}^{\mathbf{2}}$ & $\mathbf{z}_{\mathbf{2} \mathbf{j}}^{\mathbf{2}}$ & $\mathbf{z}_{\mathbf{3} \mathbf{j}}^{\mathbf{2}}$ & $\mathbf{f}_{\mathbf{1} \mathbf{j}}^{\mathbf{1}}$ & $\mathbf{f}_{\mathbf{2} \mathbf{j}}^{\mathbf{1}}$ & $\mathbf{f}_{\mathbf{3} \mathbf{j}}^{\mathbf{1}}$ & $\mathbf{f}_{\mathbf{1} \mathbf{j}}^{\mathbf{2}}$ & $\mathbf{f}_{\mathbf{2} \mathbf{j}}^{\mathbf{2}}$ & $\mathbf{y}_{\mathbf{1}, \mathbf{j}}$ & $\mathbf{y}_{\mathbf{2}, \mathbf{j}}$ \\
\hline 1 & 19 & 30 & 25 & 31 & 25 & 20 & 83 & 36 & 84 & 70 & 83 & 82 & 96 & 78 & 57 & $\mathbf{6 2}$ \\
2 & 32 & 62 & 77 & 40 & 81 & 42 & 25 & 38 & 47 & 57 & 19 & 44 & 50 & 36 & 42 & $\mathbf{3 3}$ \\
3 & 49 & 67 & 32 & 34 & 33 & 50 & 43 & 71 & 53 & 51 & 36 & 21 & 31 & 78 & 57 & $\mathbf{6 0}$ \\
4 & 56 & 71 & 51 & 23 & 29 & 14 & 81 & 53 & 73 & 43 & 19 & 18 & 82 & 14 & 29 & $\mathbf{3 1}$ \\
\hline
\end{tabular}

Table 9

Numerical results for two-stage problems of model 1 with values of $\delta \alpha=\delta \beta=0.1$

\begin{tabular}{|c|c|c|c|c|c|c|c|c|c|}
\hline \multicolumn{4}{|c|}{ Model (16) } & \multicolumn{3}{|l|}{ Model (17) } & \multicolumn{3}{|l|}{ Model (18) } \\
\hline DMU & $\begin{array}{c}\text { Stage } 1 \\
\text { efficiency }\end{array}$ & $\begin{array}{c}\text { Stage } 2 \\
\text { efficiency }\end{array}$ & $\begin{array}{c}\text { Stage } 3 \\
\text { efficiency }\end{array}$ & $\begin{array}{c}\text { Stage } 1 \\
\text { efficiency }\end{array}$ & $\begin{array}{c}\text { Stage } 2 \\
\text { efficiency }\end{array}$ & $\begin{array}{c}\text { Stage } 3 \\
\text { efficiency }\end{array}$ & $\begin{array}{c}\text { Stage } 1 \\
\text { efficiency }\end{array}$ & $\begin{array}{c}\text { Stage } 2 \\
\text { efficiency }\end{array}$ & $\begin{array}{c}\text { Stage } 3 \\
\text { efficiency }\end{array}$ \\
\hline 1 & 1.0000 & 0.7000 & 1.0000 & 0.2000 & 0.9000 & 1.0000 & 1.0000 & 0.7000 & 1.0000 \\
\hline 2 & 1.0000 & 0.2000 & 1.0000 & 0.6000 & 0.7000 & 0.9662 & 0.6000 & 0.7000 & 0.9662 \\
\hline 3 & 1.0000 & 0.2000 & 1.0000 & 0.5000 & 0.7000 & 1.0000 & 0.5000 & 0.7000 & 1.0000 \\
\hline 4 & 0.3000 & 0.3000 & 0.9829 & 0.3000 & 0.3000 & 0.9829 & 0.3000 & 0.3000 & 0.9829 \\
\hline
\end{tabular}

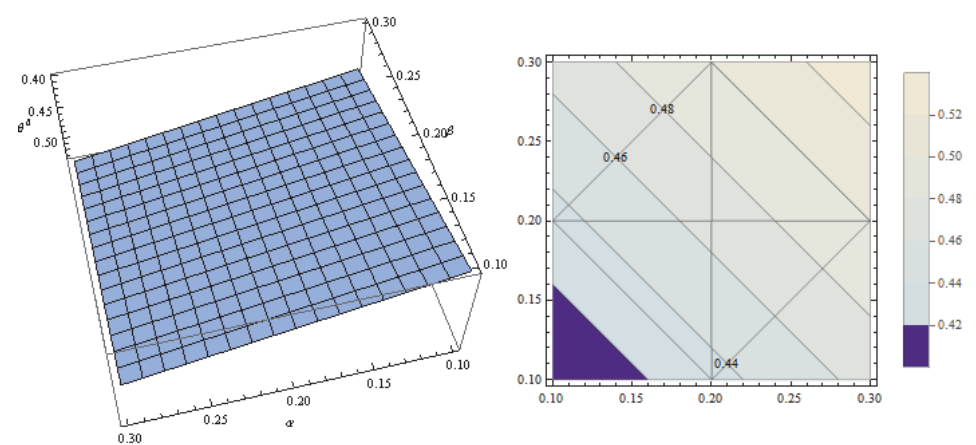

Fig. 3. Contour plot and numerical results for model 2 


\section{Conclusion}

In this paper, two types of three-stage data network envelopment analysis model with series structure with feedback were studied. The models examined have one feedback and two feedback. In modeling these problems, one-stage and two-stage programming problems have been used in order to evaluate the efficiency. At first, the efficiency of the total system was studied without considering the importance of the sub-sections and a nonlinear programming problem was introduced with considering the total efficiency average. Then, by considering the relationships between the stages, a two-stage programming problem was presented with regard to the importance of each stage. The presented programming problems have nonlinear structure. This nonlinear programming model was transformed into a linear programming model by using the coordinate transformation method and assuming constant some of the variables, and by applying step by step motion method, the problems were solved. Finally, by presenting some test problems, the theory section of the numerical results was used in practice and the results were presented in the form of the table and graph. Also, the results of the linearization were compared with the numerical results obtained from the interior functions of MATLAB.

\section{References}

Adam Shariff Adli, A., Nur Izzati, J., Maznah, M. K., Kamal, M., \& Nawawi, M. (2017). A two stage data envelopment analysis model with undesirable output. In Journal of Physics: Conference Series, $890(1), 1-5$.

Ahlatcioglu, M., \& Tiryaki, F. (2007). Interactive fuzzy programming for decentralized two-level linear fractional programming (DTLLFP) problems. Omega, 35(4), 432-450.

Amirteimoori, A., Toloie-Eshlaghi, A., \& Homayoonfar, M. (2014). Efficiency measurement in TwoStage network structures considering undesirable outputs. International Journal of Industrial Mathematics, 6(1), 65-71.

Ashrafi, A., Jaafar, A. B., \& Lee, L. S. (2010). Two-stage data envelopment analysis: An enhanced Russell measure model. In International Conference on Business and Economics Research, 1, 3438.

Ashrafi, A., Jaafar, A. B., Lee, L. S., \& Abu Bakar, M. R. (2011). A Slacks-Based Measure of Efficiency in Two-Stage Data Envelopment Analysis. International Journal of Mathematical Analysis, 5(29), 1435-1444.

Bialas, W. F., \& Karwan, M. H. (1984). Two-level linear programming. Management Science, 30(8), 1004-1020.

Candler, W., \& Townsley, R. (1982). A linear two-level programming problem. Computers \& Operations Research, 9(1), 59-76.

Charnes, A. and Cooper, W.W. (1962) Programming with linear fractional functionals. Naval Research Logistics Quarterly, 9(3-4), 181-186.

Cooper, W. W., Seiford, L. M., \& Zhu, J. (2004). Data envelopment analysis. In Handbook on data envelopment analysis (pp. 1-39). Springer, Boston, MA.

Dantzig, G. (2016). Linear programming and extensions. Princeton university press.

Despotis, D. K., Sotiros, D., \& Koronakos, G. (2016). A network DEA approach for series multi-stage processes. Omega, 61(2016), 35-48.

Drury, C. (2006). Cost and management accounting: an introduction. Cengage Learning EMEA.

Ebrahimnejad, A., Tavana, M., Lotfi, F. H., Shahverdi, R., \& Yousefpour, M. (2014). A three-stage data envelopment analysis model with application to banking industry. Measurement, 49(2014), 308-319.

Fernandes, F. D. S., Stasinakis, C., \& Bardarova, V. (2018). Two-stage DEA-Truncated Regression: Application in banking efficiency and financial development. Expert Systems with Applications, 96, 284-301.

Halkos, G. E., Tzeremes, N. G., \& Kourtzidis, S. A. (2014). A unified classification of two-stage DEA models. Surveys in Operations Research and Management Science, 19(1), 1-16. 
Kao, C. (2006). Interval efficiency measures in data envelopment analysis with imprecise data. European Journal of Operational Research, 174(2), 1087-1099.

Kao, C. (2014). Network data envelopment analysis: A review. European Journal of Operational Research, 239(1), 1-16.

Kao, C., \& Liu, S. T. (2003). A mathematical programming approach to fuzzy efficiency ranking. International Journal of Production Economics, 86(2), 145-154.

Kao. C. (2017). Network Data Envelopment Analysis: Foundations and Extensions. Springer.

Li, H., Chen, C., Cook, W. D., Zhang, J., \& Zhu, J. (2018). Two-stage network DEA: Who is the leader?. Omega, 74, 15-19.

Li, X. (2017). A fair evaluation of certain stage in a two-stage structure: revisiting the typical two-stage DEA approaches. Omega, 68, 155-167.

Liang, L., Li, Z. Q., Cook, W. D., \& Zhu, J. (2011). Data envelopment analysis efficiency in two-stage networks with feedback. IIE Transactions, 43(5), 309-322.

Lim, S., \& Zhu, J. (2018). Primal-dual correspondence and frontier projections in two-stage network DEA models. Omega. https://doi.org/10.1016/j.omega.2018.06.005

Nabahat, M. (2015). Two-Stage DEA with Fuzzy Data. International Journal of Applied, 5(1), 51-61.

Sakawa, M., \& Nishizaki, I. (2001). Interactive fuzzy programming for two-level linear fractional programming problems. Fuzzy Sets and Systems, 119(1), 31-40.

Sakawa, M., \& Nishizaki, I. (2002). Interactive fuzzy programming for two-level nonconvex programming problems with fuzzy parameters through genetic algorithms. Fuzzy Sets and Systems, 127(2), 185-197.

Seiford, L. M., \& Zhu, J. (1999). Profitability and marketability of the top 55 US commercial banks. Management Science, 45(9), 1270-1288.

Shenoy, G. V. (2007). Linear programming: methods and applications. New Age International.

Shewell, P., \& Migiro, S. (2016). Data envelopment analysis in performance measurement: A critical analysis of the literature. Problems and Perspectives in Management, 14(3), 705-713.

Tavana, M., \& Khalili-Damghani, K. (2014). A new two-stage Stackelberg fuzzy data envelopment analysis model. Measurement, 53, 277-296.

Wang, C. H., Gopal, R. D., \& Zionts, S. (1997). Use of data envelopment analysis in assessing information technology impact on firm performance. Annals of operations Research, 73, 191-213.

Wanke, P., \& Barros, C. (2014). Two-stage DEA: An application to major Brazilian banks. Expert Systems with Applications, 41(5), 2337-2344.

Xie, F., Butt, M. M., \& Li, Z. (2017). A feasible flow-based iterative algorithm for the two-level hierarchical time minimization transportation problem. Computers \& Operations Research, 86, 124 139.

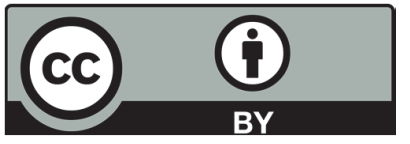

(C) 2019 by the authors; licensee Growing Science, Canada. This is an open access article distributed under the terms and conditions of the Creative Commons Attribution (CC-BY) license (http://creativecommons.org/licenses/by/4.0/). 